\title{
Des gènes qui ne tiennent pas en place !
}

\author{
Prix Nobel de médecine 1987 : Susumu Tonegawa
}

L'accumulation des renseignements sur la structure des anticorps et leur composition antigénique créait, dans les années 60 , un paradoxe difficile à expliquer sur une base génétique classique : l'extraordinaire diversité des spécificités possibles des anticorps, due à la variabilité de structure de la partie $\mathrm{N}$ terminale des chaînes d'immunoglobuline, suggérait qu'il existait un grand nombre de "gènes" codant pour les anticorps. A l'inverse, la monotonie structurale de la partie $\mathrm{C}$ terminale d'un type donné d'immunoglobuline et la ségrégation mendelienne de déterminants " allotypiques " correspondant à des variations (d'un seul acide animé) de la séquence de cette région suggérait qu'elle était codée par un seul gène possédant plusieurs allèles. Pour "réconcilier " ces deux données contradictoires, Dreyer et Bennet avaient proposé, en 1965, que les parties constantes et les parties variables des anticorps étaient codées par des " gènes " différents qui se réarrangeaient dans les lymphocytes B au cours de leur différenciation [1]. Ces auteurs supposaient l'existence de nombreux gènes de parties variables et d'un seul gène par type de partie constante.

Cette théorie impliquait, près de 10 ans avant que ces notions ne fussent démontrées, que des gènes pouvaient être morcelés... et se réarranger au cours de la différenciation de certaines cellules somatiques, deux notions parfaitement hérétiques à l'époque.

Il fallait attendre, vers 1973, l'irruption des techniques des recombinants d'ADN (d'abord les enzymes de restriction, puis les vecteurs de clonage) pour que fut définitivement démontré le caractère prophétique de l'hypothèse de Dreyer et Benett. Susumu Tone- vaillant à l'institut d'immunologie de Bâle (Suisse), démontrèrent en 1976 que l'arrangement des gènes d'immunoglobuline n'était pas le même dans l'ADN d'embryon de souris et dans celui de plasmocytome de souris (prolifération de cellules B produisant des anticorps). Ces auteurs coupèrent les deux types d'ADN avec l'enzyme Bam H1, séparèrent les fragments par migration électrophonétique dans un gel d'agarose, puis les hybridèrent avec un ARN de la chaîne $x$ de l'immunoglobuline produite par les cellules myélomateuses, marqué à l'iode 125 .

Les diagrammes d'hybridation, nettement différents pour les fragments clivés à partir des deux types d'ADN, prouvaient qu'il y avait un réarrangement de ces fragments génétiques dans les cellules produisant des anticorps.

Puis, grâce à des moyens apparaissant " héroïques " aujourd'hui, Tonegawa parvint à cloner un fragment du gène réarrangé [3] et à démontrer que le segment codant pour la partie variable et celui codant pour la partie constante étaient séparés par un intron [4]. Dans les cellules embryonnaires, cependant, les " segments variables" semblaient considérablement plus éloignés des parties constantes que dans les cellules productives.

Enfin... pour cette partie de l'aventure, Tonegawa collaborait avec Allan Maxam et Walter Gilbert (d'autres prix Nobel !) pour déterminer la séquence nucléotidique d'un segment codant pour la région variable d'un gène non réarrangé [5]. Cette étude démontrait que, avant tout réarrangement, ce segment variable codait pour toute l'information (parties hyper-variables et plus conservées) d'une partie variable d'anticorps. Par la suite, Susumu Tonegawa poursuivit son travail de dissection des gènes d'immunoglobuline et de description des mécanismes de leur réarrangement. Après son installation au MIT (Massasuchets institute of technology, Cambridge, Massasuchets, USA), il s'engageait, avec d'autres, dans une nouvelle aventure... celle du récepteur pour l'antigène des lymphocytes $\mathrm{T}$.

Sa découverte du réarrangement des gènes d'immunoglobuline en 1976, un des premiers très grands succès à l'aube du génie génétique, constitue une date capitale de l'histoire de la biologie : elle démontrait comment, ici dans des cellules somatiques se différenciant, la nature pouvait créer de nouvelles spécificités en combinant de façon variable des éléments génétiques préexistant.

Ce mécanisme de création de diversité, les lecteurs de médecine/sciences le savent, est en fait de portée générale; il se retrouve dans l'évolution (phénomène "d'exon shuffling", $m / s \quad n^{\circ} 7$, (suppl.), vol. 2, p. 15) et dans la constitution des gènes fonctionnels codant pour les sous-unités du récepteur des lymphocytes $\mathrm{T}$.

A. $\mathbf{K}$.

1. Dreyer WJ, Bennett JC. The molecular basis of antibody formation : a paradox. Proc Natl Acad Sci USA $1965 ; 54: 864-9$.

2. Hozumi N, Tonegawa S. Evidence for somatic rearrangement of immunoglobulin genes coding for variable and constant regions. Proc Natl Acad Sci USA $1976 ; 73$ : 3628-32. 3. Tonegawa S, Brack C, Hozumi N, Schuller $\mathbf{R}$. Cloning of an immunoglobulin variable region gene from mouse embryo. Proc Natl Acad Sci USA $1977 ; 74: 3518-22$.

4. Brack C, Tonegawa S. Variable and constant parts of the immunoglobulin light chain gene of a mouse myeloma are 1250 non translated bases apart. Proc Natl Acad Sci USA $1977 ; 74 ; 5652-6$.

5. Tonegawa S, Maxam AM, Tizard R, Bernard $O$, Gibert $N$. Sequence of a mouse germline gene for a variable region of an immunoglobulin light chain. Proc Natl Acad Sci USA $1978 ; 75: 1485-9$. 